\title{
Costs and quality of life in multiple sclerosis in The Netherlands
}

\author{
Gisela Kobelt · Jenny Berg · Peter Lindgren • \\ Bert Anten · Mattias Ekman · Peter J. H. Jongen · \\ Chris Polman $\cdot$ Bernard Uitdehaag
}

Published online: 22 August 2007

(C) Springer-Verlag 2007

\section{Erratum to: Eur J Health Econ (2006) 7:S55-S64 DOI 10.1007/s10198-006-0378-6}

The original version of this article unfortunately contained a mistake. Several authors were missing in the HTML version of this article. The correct information is given below.

Gisela Kobelt $^{1,2}(\bowtie)$, Jenny Berg ${ }^{3}$, Peter Lindgren ${ }^{3}$, Bert Anten ${ }^{4}$, Mattias Ekman ${ }^{3}$, Peter J. H. Jongen ${ }^{5}$, Chris Polman ${ }^{6}$, Bernard Uitdehaag ${ }^{6}$
${ }^{1}$ Lund University, Lund, Sweden

${ }^{2}$ European Health Economics, Spéracèdes, France ${ }^{3}$ Stockholm Health Economics, Stockholm, Sweden ${ }^{4}$ Maaslandziekenhuis, Sittard, The Netherlands ${ }^{5}$ MS Centre, Nijmegen, The Netherlands ${ }^{6}$ Free University Medical Centre, Amsterdam, The Netherlands

The online version of the original article can be found under doi:10.1007/s10198-006-0378-6.

G. Kobelt

Lund University, Lund, Sweden

G. Kobelt $(\bowtie)$

European Health Economics, Spéracèdes, France

e-mail: gisela.kobelt@he-europe.com

J. Berg $\cdot$ P. Lindgren $\cdot$ M. Ekman

Stockholm Health Economics, Stockholm, Sweden

B. Anten

Maaslandziekenhuis, Sittard, The Netherlands

P. J. H. Jongen

MS Centre, Nijmegen, The Netherlands

C. Polman $\cdot$ B. Uitdehaag

Free University Medical Centre, Amsterdam, The Netherlands 